Cahiers balkaniques

Balkaniques

$41 \mid 2013$

Evliyâ Çelebi et l'Europe

\title{
Pratique médicale européenne dans le Seyahatnâme d'Evliyâ Çelebi
}

European medical science in Evliyâ Çelebi's Seyahatnâme

Luca Berardi

\section{OpenEdition}

Journals

Édition électronique

URL : https://journals.openedition.org/ceb/4010

DOI : $10.4000 /$ ceb.4010

ISSN : 2261-4184

Éditeur

INALCO

Édition imprimée

Pagination : 145-156

ISBN : 978-2-85831-205-4

ISSN : 0290-7402

\section{Référence électronique}

Luca Berardi, « Pratique médicale européenne dans le Seyahatnâme d'Evliyâ Celebi », Cahiers

balkaniques [En ligne], 41 | 2013, mis en ligne le 20 mai 2013, consulté le 06 juillet 2021. URL : http:// journals.openedition.org/ceb/4010; DOI : https://doi.org/10.4000/ceb.4010

Ce document a été généré automatiquement le 6 juillet 2021.

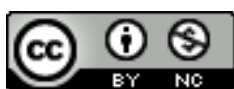

Cahiers balkaniques est mis à disposition selon les termes de la Licence Creative Commons Attribution - Pas d'Utilisation Commerciale 4.0 International. 


\title{
Pratique médicale européenne dans le Seyahatnâme d'Evliyâ Çelebi
}

European medical science in Evliyâ Çelebi's Seyahatnâme

\author{
Luca Berardi
}

Ils sont tous Arabes, parce que la science de la médecine a été donnée à la race arabe. [...] Ensuite est arrivé l'intérêt des Grecs et des Francs. Les médecins grecs et francs ont atteint la réputation mais ils ont besoin des Arabes ${ }^{1}$.

1 Parmi les pages les plus célèbres du Seyahatnâme, on trouve trois descriptions d'opérations chirurgicales, auxquelles Evliyâ Çelebi raconte avoir assisté pendant son séjour à Vienne, quand il suivait la mission diplomatique de Kara Mehmed Paşa auprès du roi Léopold $\mathrm{I}^{\text {er }}$, en $1665^{2}$.

2 La première opération décrite est la réduction d'une fracture crânienne qui a eu lieu, selon Evliyâ, dans une petite clinique royale annexée à l'église Saint Étienne à Vienne. Le patient, qui faisait partie de la maison royale, avait été blessé pendant la bataille de Raab. Il a été soumis à une trépanation du crâne pour en extraire le projectile, puis la suture a été cousue avec des mâchoires de fourmis. L'opération a réussi et le patient est sorti de l'hôpital quinze jours plus tard.

3 Evliyâ décrit ensuite une deuxième intervention faite par un chirurgien allemand à Monastir. Dans ce cas, il s'agissait d'un traitement de drainage sur un homme atteint d'une grave forme d'hydropisie, très probablement causée par une forte décompensation hépatique. Là aussi, les soins réussissent et le patient peut reprendre son voyage.

4 La troisième intervention a lieu dans le laboratoire d'un maître chirurgien (cerrâh dükkânı), en réalité la boutique d'un barbier-chirurgien traditionnel. Elle consiste en l'extraction, avec cautérisation et réimplantation autologue, d'une dent malade. Cette même dent, après avoir été extraite et traitée adéquatement, est réimplantée au 
patient. Evliyâ est particulièrement frappé par l'habileté du docteur et il en profite pour se faire soigner les dents lui aussi.

5 Evliyâ s'attarde encore sur une longue description des cantines pour les pauvres de la ville, qui, pour un observateur habitué comme il l'était au système des vakıf-imârets ottoman, représentaient une partie intégrante du système de charité et de santé publique d'un pays.

6 Par le passé, beaucoup a déjà été dit sur ces interventions chirurgicales. En particulier, considérant que le réalisme d'Evliyâ décline au fur et à mesure de son éloignement du centre de l'Empire ottoman ${ }^{3}$, on a cherché à établir ce qu'il y avait de vraisemblable dans sa description. La confrontation avec l'état de la chirurgie en Europe au XVII siècle offre une certaine base factuelle aux descriptions d'Evliyâ: ainsi, les détails apparemment les plus fantastiques de ses descriptions, comme les mâchoires des fourmis utilisées pour suturer les blessures, trouvent une confirmation dans la littérature et dans la pratique médicales de l'époque. En outre, il apparaît que certaines de ces pratiques étaient présentes également dans la culture médicale islamique. Donc, on peut émettre l'hypothèse qu'Evliyâ a pu connaître ces techniques dans le monde ottoman même.

7 Nous croyons inutile de nous attarder plus longtemps ici sur les données factuelles de ces récits, déjà abondamment discutées ${ }^{4}$. Nous pouvons les synthétiser en disant que le récit d'Evliyâ entre dans la catégorie du "plus vrai que nature ». Nous ne pouvons savoir avec certitude s'il a assisté réellement à ce qu'il décrit ; toutefois, son récit nous offre une description cohérente avec l'état de l'art de la chirurgie européenne. Il fournit une description très précise des détails anatomiques, mais, dans ce cadre remarquable de réalisme, il insère des éléments fantastiques. Un détail peut apparaître significatif : à l'occasion de la troisième intervention, après que le chirurgien ait extrait la dent et cautérisé les racines avec des coutures en laiton chauffé au rouge, Evliyâ affirme qu'il voit sortir des gencives de tout petits vers à tête noire ${ }^{5}$. Il faut se demander pourquoi, dans un récit extrêmement plausible, il insère un détail si nettement fantastique. À ce propos, on a remarqué que dans la tradition médicale islamique du Moyen Âge, beaucoup de maladies dentaires avaient été attribuées à la présence de larves à la base des dents. Evliyâ, peut-être influencé par cette croyance, aurait confondu la matière infectée avec de petites larves ${ }^{6}$. Dans ce cas, l'élément fantastique n'aurait pas été inséré volontairement dans la description, il s'agirait plutôt de la conséquence d'un malentendu culturel.

8 Cet élément introduit l'objet réel de cette communication. On voudrait proposer certaines observations sur la façon dont Evliyâ, un représentant de l'élite ottomane urbaine $\mathrm{du} \mathrm{XVII}^{\mathrm{e}}$ siècle, comprend les informations sur les sciences médicales européennes. Comment perçoit-il et interprète-t-il ce qu'il observe? En d'autres termes, quels facteurs culturels conditionnent son récit? Il faudra avant tout reconstruire la perception de la médecine qu'avait Evliyâ et pour ce faire, se pencher à nouveau sur le Seyahatnâme, qui offre de nombreuses informations à cet égard.

\section{La maladie et ses causes}

9 Le système médical ottoman était l'expression plutôt fidèle du caractère composite de la culture ottomane elle-même. Miri Shefer-Mossensohn a décrit la coexistence de trois 
" sous-systèmes ", chacun caractérisé par des pratiques particulières, et ayant une base de légitimité différente :

- la médecine mécaniciste de la tradition galénique, d'origine grecque, basée sur la notion d'équilibre entre les quatre humeurs du corps humain (le sang, la bile jaune, l'atrabile et la pituite) ;

- la médecine religieuse, ou médecine prophétique (țıbb-ı nebevî), qui tenait son autorité de la sunna du Prophète ;

- enfin, la médecine populaire et magique, dans laquelle convergeaient différentes traditions, du chamanisme d'Asie centrale à l'héritage anatolien pré ottoman. Cette dernière forme, contrairement aux deux précédentes, n'avait pas de tradition écrite et basait sa propre légitimation non pas sur une autorité scientifique, mais sur le consentement populaire ${ }^{7}$.

Dans ce vaste « espace » médical s'exerçait l'action de nombreux opérateurs depuis les docteurs professionnels jusqu'aux charlatans et aux sorciers.

Bien que la médecine mécaniciste-humoraliste représentât la médecine cultivée et presque officielle puisqu'elle était pratiquée à la Cour, on peut affirmer qu'aucun de ces trois systèmes n'avait l'hégémonie. De plus, les trois traditions n'étaient pas forcément en conflit les unes avec les autres. Elles étaient vues plutôt comme complémentaires. Les docteurs et les patients adoptaient, parfois inconsciemment, l'une ou l'autre théorie. Les frontières des différents domaines médicaux ottomans n'étaient donc pas imperméables et se confondaient souvent en se croisant $^{8}$.

Dans ce domaine, Evliyâ Çelebi apparaît comme un Ottoman "typique", pour reprendre la définition de Robert Dankoff. Ceci est particulièrement évident dans ses réflexions à propos de l'origine des maladies. Dans un chapitre du $\mathrm{x}^{\mathrm{e}}$ livre du Seyahatnâme consacré au climat en Égypte, Evliyâ souligne l'influence des facteurs environnementaux sur la santé des habitants de la région. Il relie l'apparition de différentes maladies au climat, à certains vents et, en général, à des conditions environnementales malsaines. Ce sont des concepts qui nous ramènent à la médecine galénique, donc à un système médical naturaliste.

Quelques lignes plus loin cependant, on trouve l'opinion, attribuée aux oulémas, selon laquelle l'éléphantiasis, particulièrement répandue au Caire, atteindrait en particulier les Yazidis, et qu'elle serait la conséquence de l'outrage infligé par leurs ancêtres au corps de l'imam Hüseyn'. La cause de la maladie est donc expliquée cette fois-ci en termes de punition divine, en cohérence avec les canons de la médecine prophétique. En d'autres termes, elle est provoquée par un agent étiologique typique d'un système médical personnaliste, dans lequel la maladie est causée par une intervention surnaturelle délibérée, par exemple l'action d'un agent intelligent. Comme on le voit, l'idée de médecine ici énoncée utilise deux conceptions étiologiques antithétiques, qui ne sont toutefois pas en contradiction dans la perception ottomane ${ }^{10}$.

14 À Raguse, Evliyâ Çelebi fut obligé à une étape forcée de trois jours au lazaret (nażarete) de Bândışka. À cette occasion, il entra en contact avec une pratique de la prophylaxie européenne, la quarantaine. Toutefois il ne semble pas croire à l'utilité de ce système pour prévenir la diffusion de la peste. En particulier, il juge « vaines croyances » $\left(z u^{\prime} m-l\right.$ bâț̣ller) la confiance dans la prophylaxie appliquée aux marchandises dangereuses, comme la laine et les tissus, pour lesquelles étaient prévues une fumigation et des saupoudrages de vinaigre. 
On peut imaginer que ce jugement était le résultat, au moins partiellement, d'une hostilité envers une limitation qui empêchait l'échange des marchandises et gênait les marchands qui venaient de l'Empire ottoman et, n'étant pas en mesure de produire les documents sanitaires exigés, étaient obligés de subir de longues détentions qui causaient des dommages évidents au commerce ${ }^{11}$. En même temps, ce jugement pourrait être représentatif de la manière dont Evliyâ concevait la pratique médicale européenne.

Les bailes vénitiens et les différents voyageurs européens contemporains s'étonnaient de la complète insouciance des habitants d'Istanbul à l'égard des précautions les plus élémentaires contre la contagion. Ces observateurs européens faisaient entrer ces conduites dans les conséquences du «fatalisme oriental», en l'attribuant à une confiance absolue dans le « destin ». Au contraire, il est largement prouvé que dans le monde ottoman, le sens commun conduisait à éviter les lieux où se manifestait une épidémie. En outre, l'utilité et la légitimité religieuse de la fuite étaient théorisées dans les traités sur la peste, produits principalement aux $\mathrm{XVI}^{\mathrm{e}}$ et $\mathrm{XVII}^{\mathrm{e}}$ siècles $^{12}$. Ainsi, l'attitude ottomane envers la maladie n'était pas celle de l'acceptation fataliste ${ }^{13}$. Toutefois, bien qu'une discussion sur la contagion ait existé, les différentes traditions médicales ottomanes n'avaient pas développé de "théorie de la contagion ", c'est-àdire qu'elles n'avaient pas établi une idée sur la manière dont les hommes ou les choses pouvaient être le véhicule de diffusion de la maladie ${ }^{14}$. En fait, dans le monde ottoman, la théorie principale sur la diffusion de la maladie s'en tenait à la conception galénique du miasme, c'est-à-dire à une cause complètement environnementale. La peste s'enracine là où les conditions du terrain et du climat en favorisent l'apparition. La médecine prophétique, au contraire, prenant en considération de nombreux hadîs, reliait l'origine de la peste à la corruption morale et aux djinns, ou à l'influence astrale, qui seraient les instruments du châtiment divin.

D'après différents passages du Seyahatnâme, il semblerait que Evliyâ avait une notion intuitive de la contagion. Il relie - par exemple - la syphilis au contact cutané15. Toutefois, quand à Raguse, il est témoin d'une pratique sanitaire européenne comme la quarantaine, il utilise les schémas propres à sa culture médicale et rejette cette pratique comme inutile.

\section{Rôle de la chirurgie}

18 Il faut aussi considérer un autre aspect: les pratiques médicales européennes que Evliyâ décrit font partie de la médecine thérapeutique et il s'attarde sur une forme particulière de thérapie, l'intervention chirurgicale.

19 La chirurgie était vastement attestée dans les textes médicaux ottomans. Le médecin anatolien şerefeddîn Sabuncuoğlu a écrit son traité sur la chirurgie, le Cerrahîyet-i İlHânîye (La chirurgie Ilkhanide) en 1465, deux siècles donc avant la visite à Vienne d'Evliyâ Çelebi ${ }^{16}$. Aujourd'hui, ce traité est l'un des traités médicaux ottomans les plus connus. Il s'agit d'une traduction en turc de l'encyclopédie médicale $\mathrm{du} \mathrm{XI}^{\mathrm{e}}$ siècle al-Tașrîf li-man 'ajaza 'an al-ta'lîf d'Abu'l-Qâsim al-Zahrâwî, connu dans les sources occidentales comme Abulcasis, que le chirurgien turc a partiellement enrichie avec des expériences faites sur le terrain. 
Le troisième chapitre de l'ouvrage est consacré aux fractures et aux luxations. Dans la deuxième section - qui a été traduite assez fidèlement du texte d'Abû'l-Qâsim ${ }^{17}$ Sabuncuoğlu décrit les différentes typologies de fracture crânienne, leurs causes (coups d'épée, collisions, chutes), le diagnostic et les éventuels traitements chirurgicaux. L'une de ces interventions, que la majorité des chirurgiens seraient quoi qu'il en soit incapables de réaliser, ressemble grandement à l'opération décrite par Evliyâ.

Si la fracture touche la membrane qui couvre le cerveau (par ex. les méninges) et que les os sont brisés en de nombreux fragments, il est nécessaire de couper et d'extraire ces os. Décrivons alors cette [opération]. Il est nécessaire de graver avec différents instruments. La procédure pour extraire ces fragments d'os - si Dieu le veut - est la suivante : tu vas raser les cheveux du malade et tu vas découvrir l'os de la blessure, en suivant le plus possible la forme de la blessure. Tu ne dois pas effrayer le malade [...]. Après ceci, tu continueras à creuser l'os et à en extraire les fragments [...]. Si l'os du crâne est dur, tu vas faire des trous avec le trépan tout le long du périmètre de la zone de la fracture. [...] Chaque fois que tu vas commencer un trou, tu placeras le trépan à côté de la zone de la blessure et tu le tourneras avec la main jusqu'à ce que le trou soit achevé et la pointe du trépan ait traversé l'os. Ainsi tu le soulèveras et tu le placeras sur un autre point et aussi dans ce cas tu vas procéder de la même façon [en faisant attention que] l'éloignement entre les deux trous soit égal à l'épaisseur d'un poinçon, ou un tout petit peu plus grande. Après tu couperas l'espace entre les deux trous avec les ciseaux appropriés parmi ceux qu'on vient de décrire ci-dessus.

Après avoir tout coupé de cette façon, pour ce qui est possible, tu vas enlever [l'os] avec les mains ou avec un instrument tel qu'un forceps ou une pince souple. Pendant que tu réalises cette opération, fais attention à ce que ni le ciseau ni le trépan ne touchent la membrane. Ainsi tu vas racler avec le ciseau la partie pour éliminer la surface raboteuse. [...] S'il reste des petits fragments d'os brisé, tu vas les extraire avec un instrument approprié. Ensuite tu vas traiter la blessure avec des onguents qui sont étudiés dans le dernier chapitre de ce livre ${ }^{18}$.

21 Les différences entre l'opération de Sabuncuoğlu et celle qui se tint à Vienne semblent n'être qu'une conséquence de la différence de niveau technologique. Les instruments du chirurgien anatolien, des trépans, ciseaux (mıkța) et forceps (kelpeteyn), avaient été remplacés en Europe au $\mathrm{XVII}^{\mathrm{e}}$ siècle par la tréphine, plus raffinée, "la pince de fer » (demir mengane) décrite par Evliyâ.

Donc, sur le plan théorique, les Ottomans avaient les connaissances chirurgicales nécessaires pour réaliser une opération comme celle de Vienne. En réalité, il faut supposer que, dans ce domaine, il y avait un écart remarquable entre la théorie et son application ${ }^{19}$.

Une grande partie des manuels médicaux était adressée aux oulémas, qui les commandaient pour perfectionner une connaissance de la médecine la plupart du temps exclusivement théorique. Les hekîms eux-mêmes, les docteurs ayant une formation académique, observant la théorie galénique, adoptaient surtout, ou seulement, des thérapies non invasives, destinées à rétablir l'équilibre des humeurs corporelles du patient. Donc, il faut penser que beaucoup des traitements chirurgicaux présents dans ces traités, bien que complexes, ne faisaient pas partie de l'activité médicale ottomane commune. La chirurgie ottomane se limitait principalement aux traitements de phlébotomie (hicâma) et de cautérisation (dag்), pratiqués par des chirurgiens professionnels ainsi que par des barbiers chirurgiens privés ${ }^{20}$. Dans ces domaines, les chirurgiens ottomans avaient atteint un niveau technique très élevé, 
supérieur même à celui des Européens, comme nous le dit Prospero Alpini, le docteur italien qui visita l'Égypte à la fin du XvI ${ }^{\mathrm{e}}$ siècle.

En général, l'attitude des Ottomans envers la chirurgie était celle d'une extrême méfiance, une méfiance qui allait aussi à l'encontre des professionnels et des semiprofessionnels qui travaillaient dans ce domaine. Le grand nombre de complications qui suivaient les opérations et le taux élevé de mortalité impliquaient que la chirurgie invasive fût une extrema ratio. Elle était pratiquée dans les cas sans espoir, en l'absence d'alternatives.

À cet égard, dans le Seyahatnâme, on trouve un exemple assez éloquent. Evliyâ nous raconte que pendant que son oncle Melek Ahmed Paşa était gouverneur de Özü, un abcès se gonfla dans un coin de son cou. Bien qu'il ait consulté de nombreux docteurs, phlébotomes et chirurgiens, la condition du pacha s'aggrava au point qu'on pensa qu'il allait mourir. Seule l'interprétation d'un rêve, selon les règles de l'ilm al-ta'bîr, l'oniromancie islamique, le convainquit de se tourner vers un traitement chirurgical, une incision, qui permit de nettoyer l'abcès de la matière infectée. Ceci advint, non sans qu'Evliyâ, auteur du rêve, s'opposât à l'intervention: "Vous êtes en train d'inciser votre cou. Si votre blessure s'aggrave, vous allez me mettre en difficulté. Ils me diront : "le pacha s'est fait opérer au cou à cause du rêve d'Evliyâ et il est mort". [...] Par miséricorde mon sultan, ne faites pas venir le chirurgien pour vous faire inciser l'abcès $»^{21}$.

\section{Médicaments ou intervention?}

En général, dans la hiérarchie de la médecine ottomane, la chirurgie occupait une position subalterne. En effet, dans la mentalité ottomane, on se consacrait plutôt à la préservation de la santé qu'au soin des maladies. Par conséquent la médecine préventive (himâye), avec le respect d'un régime alimentaire et des normes hygiéniques, était plus importante que la médecine thérapeutique. La pharmacologie, au sens large, se situait entre les deux domaines, prévention et soin. L'administration de produits médicaux, les sirops, les électuaires ( $\left.m a^{\imath} c u ̂ n\right)$, le thériaque (tiryâk $k$ - fârûk ), avaient la double fonction de préserver ou de rétablir l'équilibre corporel et donc la santé. Ainsi, le domaine de la pharmacologie dans le monde ottoman était très vaste. Comme l'a observé Rhoads Murphey à Istanbul, le groupe de ceux qui vendaient des produits pour la santé dépassait en quantité celui de ceux qui offraient une assistance médicale (1992).

Des descriptions des différents hôpitaux ottomans (bîmâristân ou dârü'şşifâ), dans le Seyahatnâme, on peut déduire un cadre assez clair de la pratique médicale dans ces institutions. Les soins décrits sont surtout des pratiques non-invasives qui peuvent se résumer en une alimentation adéquate, de bonnes conditions d'hygiène et l'utilisation d'électuaires et de coups de fouet pour faire recouvrer la raison aux fous ${ }^{22}$. Les hôpitaux ottomans offraient en outre des conditions environnementales favorables au bien-être des patients grâce à leur architecture, avec jardins, vasques et fontaines, et grâce à l'usage thérapeutique de la musique. L'habileté du médecin ottoman pouvait être vue dans le diagnostic qu'il était capable de faire par la prise du pouls, et dans la prescription correcte de médicaments. 
n peut savoir dans quelle mesure et de quelle manière Evliyâ Çelebi a été influencé par ces attitudes ottomanes envers la santé et la médecine lorsqu'il observait la pratique médicale européenne, en partant de son récit. Dans ce récit en effet, seul un élément est absolument fantastique : le prodige de la dent qui bouge. Evliyâ raconte qu'après avoir vu réussir la réimplantation de la dent d'un infidèle, il aurait demandé au chirurgien de soigner trois de ses dents qui étaient chancelantes, à cause d'un coup qu'il avait reçu en jouant à cirîd quelques années auparavant. Grâce à un liquide rouge aux pouvoirs miraculeux, appliqué sur la gencive, ses dents seraient redevenues solides comme le fer, au point qu'il pouvait les utiliser pour casser des noix ${ }^{23}$. Il y a un parallèle intéressant à cette anecdote dans le livre x du Seyahatnâme. Dans ce cas également, après avoir décrit les hôpitaux du Caire et la production de la thériaque, Evliyâ ajoute une anecdote pour illustrer les effets de ces panacées. Il raconte avec beaucoup de détails comment il a été guéri de la stérilité qui le tourmentait depuis plus de vingt ans, après avoir bu un extrait de serpent produit par les docteurs égyptiens ${ }^{24}$. Dans le Seyahatnâme, on trouve de nombreux épisodes similaires, où la prise de substances médicales produit une guérison prodigieuse ${ }^{25}$.

Dans les deux récits - à savoir ceux du soin des dents et de la virilité retrouvée - on retrouve une modalité de description bien connue chez Evliyâ, qui visait à conjuguer l'utile à l'agréable, en laissant place à la fantaisie ${ }^{26}$. Dans le cas présent, on peut affirmer que le soin des dents qui bougent est une pure invention littéraire. L'épisode offre, en tout cas, une indication assez claire de la manière dont il interprétait pendant qu'il observait. Evliyâ comprend mal la nature de l'intervention du dentiste à laquelle il assiste, puisqu'il attribue la réussite de la greffe aux deux liquides utilisés par le médecin, dont on peut imaginer qu'ils consistaient tout simplement en un anesthésiant et un désinfectant. Enfin, pour confirmer l'effet prodigieux du médicament, il ajoute l'anecdote dont il est le protagoniste. En d'autres termes, il amplifie le rôle du médicament, et considère de façon secondaire l'aspect purement mécanique et chirurgical du traitement, encore une fois à cause de mécanismes interprétatifs propres à la conception ottomane de la médecine.

On dispose donc de quelques éléments supplémentaires pour confirmer l'attitude d'Evliyâ envers la médecine européenne. Sa stupeur face aux techniques chirurgicales européennes a été plusieurs fois soulignée par les chercheurs. En effet, il assiste à quelque chose d'inhabituel, le recours à la chirurgie invasive étant très peu fréquent chez les docteurs ottomans du XvII ${ }^{e}$ siècle. Il s'agit d'une nouveauté, telle qu'Evliyâ est disposé à payer (mais pas plus que cinquante pièces d'or) pour apprendre les secrets des soins dentaires du maître chirurgien. Miri Shefer Mossensohn a noté avec justesse que, bien qu'un peu désarçonné par la technique européenne, Evliyâ donne l'impression que «dans l'ensemble, la tradition scientifique et clinique dont il était témoin lui était plutôt familière $\aleph^{27}$. Ceci n'implique pas forcément qu'il connaissait ces traitements, mais, plus simplement qu'il réussissait à conceptualiser facilement la nouveauté à laquelle il assistait dans un cadre de référence familier, celui de la médecine ottomane.

Il y a au moins deux raisons qui permettaient cette lecture « ottomane » de la chirurgie européenne. En premier lieu, au début de l'ère moderne les grandes innovations dans la théorie et la pratique médicale européenne n'impliquaient pas l'abandon de l'héritage hellénistique puis islamique. Comme l'observe Evliyâ lui-même, les chirurgiens viennois sont, tout comme les docteurs ottomans, des émules de Platon, d'Hippocrate, 
de Socrate, et leur science est celle d'Ibn Sinâ. Il y a aussi une autre raison qui peut être vue dans l'attitude proprement ottomane à l'égard de la santé et des soins. La médecine est conçue comme un ensemble de connaissances pluriel et surtout syncrétique. Elle réside pour l'essentiel dans la capacité à accueillir et à synthétiser des éléments antithétiques.

\section{BIBLIOGRAPHIE}

Acıduman Ahmet et Er Uygur, (2006-2009), “XVII. Yüzyıl Viyana'sında kafatası cerrahisi : Evliyâ Çelebi Seyahatnamesi'nden bir olgu", Yeni Tıp Tarihi Araştırmaları 12,13,14,15, pp. 125-133.

Dankoff Robert et Kim Sooyong, (2011) An Ottoman Traveller. Selections from the Book of Travels of Evliyâ Çelebi. Londres: Eland.

Dankoff, Robert, (2004), An Ottoman Mentality. The World of Evliyâ Çelebi. With an Afterword by Gottfried Hagen, Leiden, Boston:Brill.

Dankoff Robert, (1991), The Intimate Life of an Ottoman Statesman (Melek Ahmed Paşa (1588-1662), Albany: SUNY Press.

Kreutel Richard (1957), Im Reiche des Goldenen Apfels. Graz, Wien, Köln :Verlag Styria.

Gadelrab Sherry Sayed, (2010), “Medical Healers in Ottoman Egypt. 1517-1805”, Medical History, 54, 3, pp. 365-86.

Leiser Georges et Dols Michael, (1987), “Evliyâ Chelebi’s Description of Medicine in SeventeenthCentury Egypt, Part I: Introduction”. Sudhoffs Archiv, 71, 2, pp. 197-216.

Leiser Georges et Dols Michael (1988), “Evliyâ Chelebi's Description of Medicine in SeventeenthCentury Egypt. Part II: Text”, Sudhoffs Archiv, 72, 1, pp. 49-68.

Livingston, John William, (1977), “Evliyâ Çelebi on Surgical Operations in Vienna”, Al-Abhath, 23, pp. 223-24.

Murphey, Rhoads, (1992), “Ottoman Medicine and Transcultralism from the sixteenth Century through the eighteenth Century", Bulletin of the History of Medicine, 66, pp. 376-403.

Önler, Z. 2009. “Evliyâ Çelebi'nin Viyana'dan Aktardı̆̆ı Cerrahiye İlişkin Üç Gözlem”, In N. Tezcan (dir.). Çă̆ının Sıradışı Yazarı Evliyâ Çelebi, Istanbul: Yapı Kredi Yayınları, 293-305.

Panzac, Daniel, (1999), « Politique Sanitaire et Fixation des Frontières: L'exemple Ottoman (xVIII ${ }^{\mathrm{e}}$ XIX ${ }^{\mathrm{e}}$ siècles) », Turcica, 31, pp. 87-108.

Savage-Smith, Emile, (2000), "The Practice of Surgery in Islamic Lands: Myth and Reality", Social History of Medicine, 13, pp. 307-21.

Sabuncuoğlu, şerefeddîn, (1992) Cerrâhiyyetü'l-Hâniyye, İ. Uzel (dir.). 2 vol. Ankara:Türk Tarih Kurumu Yayınları.

Shefer-Mossensohn, Miri,(2009), Ottoman Medicine. Healing and Medical Institutions 1500-1700, Albany: SUNY Press. 
SN= Evliyâ Çelebi. 1996-2007. Evliyâ Çelebi Seyahatnamesi, I-X. Istanbul:Yapı Kredi Yayınları.

Spink M. S. et Lewis G. L. (1973), Abulcasis. On Surgery and Instruments, Londres: The Wellcome Institute of the History of Medicine.

Varlık, Nükhet, (2008), Disease and Empire: an History of Plague Epidemics in the Early Modern Ottoman Empire (1453-1600), Ph.D. non publiée. University of Chicago.

White, Sam, (2010), "Rethinking Disease in Ottoman History", International Journal of Middle East Studies, 42, pp. 549-567.

\section{NOTES}

1. Yine kavmi 'Arabdır zîrâ 'ilm-i țıb evlâd-ı 'Araba verilmişdir [...] andan Yûnânîyân ve Firenk dakîkıına vâșll olup hükemấ-yi Yûnânîyân Firenk iştihâr buldılar ammâ yine 'Araba muhtâclardır.

2. SN, VII : 108-10 ; Kreutel, 1957, 126-39 ; Livingston, 1970 ; Dankoff et Kim, 2011, 242-47.

3. Dankoff, 2004, 62.

4. Livingston, 1970 ; Acıduman et Er, 2006-2009 ; Önler, 2009.

5. Bilâ 'usret zor etmeden ve ḳanı așlâ aḳmadan bir dâne beyâż üç çatal kökli şâhâne dişi çıkarup hemân mežkûr âteş kenârına gelüp mežkûr țanbûra telleriniñ birin ateşden çıkarup dişiñ köküne ḳızmış teli șoḳunca hemân tel dişıñ iligi içinden cız cız deyerek dişiñ çürügi içinden ḳızmış talıñ ucı çıkup bir karaca başlı nahîf ü rakîk ḳurdcaġı țaşra çıkup bir tel daHi ḳızmış iken tine bir köke daHi șoḳup yine dişiñ çürügünden bir ḳurdcaġı eyle çıkup.

6. Önler, 2009, 301.

7. Shefer-Mossensohn, 2009, 21-100.

8. Murphey, 1992.

9. Ve fellâhîniñ birer ayakları țulumlar gibi olur 'ulemâ-yı Mıșır eyle hikâyet éderler kim kaç̧an kim Yezîd$i$ bî-mezîd İmâm Hüseyniñ ser-i se âdetin şama gönderüp andan zîr-i hükmünde olan Mișir sulțânına gönderdi ol daHi ser-i se'âdeti Rûm-eli meydânında Sulțân Ḥasan câmi 'i yerinde biragup İmâm Hüseyniñ başın yezîdîler püşt-i pâ ile galțân etdiler zîrâ yezîdîler ol 'așırda Mișir içre vefret üzre idiler ammâ re's-i İmâm Hüseyne depme ile her kim urdı ise urduġı ayaġı țulum gibi hâalâ Mıșırda birer ayaḳları țulum gibi olanlar ol yezîdîleriñ veled-i nâ-bekârlarından deyü 'ulemâ-yı Mıṣır böyle naḳl éderler.

10. Shefer-Mossensohn, 2009, 171-72.

11. C'est à ce traitement différent qu'apparemment fait allusion Evliyâ Çelebi: $B a$ ‘ż adamlar kırk gün oțurup durur ve aḳalli on gün ve yedi gün ve üç gün elbette ve elbette oṭurtmaları mukarrerdir.

12. Varlık, 2008, 172-204.

13. White, 2010.

14. Shefer-Mossensohn, 2009, 175-79.

15. Leiser et Dols, 1987.

16. L'écriture arabe comporte une ambiguïté dans la lecture du titre de l'ouvrage qui peut être lu aussi comme Cerrâhîyetü'l-Hânîye (La Chirurgie Impériale). Uzel, 1992.

17. Spink et Lewis, 1973, 689-711.

18. Ve eger șınık beyni üzerindeki ġışâya érişüp ve pâre yéncilmiş kemükler olsa ol kemükleri kesüp çıkarmak gerekdür biz anı vașf édelüm anı nice âletile keskmek gerekdür in-şẩallâh ol ufak kemügüñ çîkarmaġınuñ țarîk-ı 'ameli oldur kim 'alîlüñ saçını yüliyesin ve cirâhatüñ kemügini ne vechile mümkinise açasın cirâhatüñ şekiline göre ve 'alîli ḳorḳutmayasın [...] andan șoñra kemügi oymaġa ve kemügüñ pârelerin çıkarmaġa meşĝûl olasın [...] eger başuñ kemügi katıyısa ol șınuk yérüñ çevresin mișkabıla delük delük édesin [...] her vakt kim delük şürû' édesin miskkabı ol șınuk yérüñ kııragina koyup eliñ ile burasin tâ kim delük delindi miskkabuñ ucı kemügüñ ötesine geçdi andan șoñra kaldurup bir yére daHii koyasin ol yéri daHi ol resme édesin ve ammâ iki delügüñ aralıġı bir biz yog்unlıgı miḳ̂ârı ola ya bir birez artukırak ola 
andan șoñra her iki delügüñ arasını ol yukaruda gösterdügümüz mıkța'laruñ bir münâsibiyile kesesin ve cemî́sin bu resme tâkatuñ yétdükçe kesdügünden șoñra elüñile yâHûd bir âletile koparasın çift gibi ya ince kelpeteyn birle bu 'ameli işlerken mișkabı ya mıkța'ı șıfâka degürmekden șaḳınasın andan șoñra mıkța' yérlerin kazıyasın tâ kim iriligi gide [...] eger ufanan kemükden ufak pârecukları kalursa aña münasib bir âletile çıkarasın andan șoñra cirâhate mu'alece édesin şol merhemlerile kim bu kitâbuñ âHir faṣlinda gelecekdür.Sabuncuoğlu, 1992, I, 375-79; II, 172A-175B.

19. Savage-Smith, 2000.

20. Gadelrab, 2010.

21. Şimdi eñseñi yararsiñ yarañ müştedd olursa Evliyânıñ gördügü vâkıı'ayla paşa yarasın yardırup helâk oldu deyü bu hạâîi âteşe yaḳdırırsiñ [...] amân sulțânım cerrâh getirüp mâddeyi yarmañ.

$\mathrm{SN}, \mathrm{V}, 56$.

22. Par exemple, à propos du bîmârHुâne de Sultan Kalâ'ûn au Caire: 'İmâretden ța âm çıkacak mahalde cümle on iki ețibbâ telâmîzleri ile herkesiñ țabî'atine göre ța âm ve ma'âcîn edviyeler verirler ve hükemâlarıñ cür'et șâhibi cellâd-meșreb Huddâmları var anlar deli birâderleriñ ba żısına kıızılcık degenegi Hoşâbı yedirirler 'aklı başına gelir.

23. Hemân hakîir bu hâl-i ber-kemâl-i pür-șavâbı görüp cânım yerine geldi zîrâ Uyvar kal'esi altında cirîd oynarken Ḳıbleli Muștafâ Paşa dişlerime bir cirîd urup üç dâne dişlerim yerlerinden mütezelzil olmışdı hemân cerrâha gösterüp ol ân ḳırmızı șudan dişlerimiñ etlerine sürüp mežkûr gibi bir pâre taHtacıgı ıșırup șabâhadak dişlerim pulad-ı NaHşsevânîye dönüp findık ve cevz kıırmaġa liyâtli dişler olup cihân ḳadar hażz édüp cerrâha bir Kaya Sulțân yag்lig̀ı verdim.

24. Netîce zürrîyet munkațı' oldı deyü bu ḥaḳ̂r me'yûs idim tâ ki yigirmi yedi sene 'ubûr édüp bu hakâir Mıșira gelüp bu Kalầ'ûn tîmârHânesinde bu fârûk güni iki fincân yılan maṣlûkası yag̉ ile içdügim géce biri biri ardı șira iki kerre iḥtilâm oldum 'ale'ș-ṣabâh yine tîmârHâneye varup hekîm başıya ser-güzeșt [ü] serencâmım naḳl etdim on vakîye yılan maṣlûkası ve bir hokkka zeyt ile mülemma' yılan yag̉ı vérdi beş altı gün yılan mașlûkasın isti 'mâl etdim ol kadar ten-dürüst oldum kim laḥm [u] şaḥm üzre findık ḳırılırdı ol kadar seng-ten oldum ve nice nefi 'lerin daHi müşâhede etdim.

25. Dankoff, 2004, 118-19.

26. Ibid., 153-84.

27. "[O]n the whole, the scientific and clinical tradition he saw was quite familiar to him". Shefer-Mossensohn, 2009, 55.

\section{RÉSUMÉS}

Parmi les pages célèbres du Seyahatnâme, on trouve trois descriptions d'opérations chirurgicales, auxquelles Evliyâ Çelebi raconte avoir assisté pendant son séjour à Vienne. On voudrait proposer ici certaines observations sur la façon dont Evliyâ, un représentant de l'élite ottomane urbaine du $\mathrm{XVII}^{\mathrm{e}}$ siècle, comprend les informations sur les sciences médicales européennes. Comment interprète-t-il ce qu'il observe? Quels facteurs culturels conditionnent son récit?

Among the famous pages of the Seyahatnâme, one finds three descriptions of surgeries to which he has been assisting - so Evliyâ wrote - during his stay in Vienna. We would here propose a few notes on the way Evliyâ, a representative of the 17th century urban ottoman social top, understands the informations about the european medical science. How does he interpret what he is seeing? Which cultural factors are influencing his story? 


\section{INDEX}

Thèmes : Histoire, Histoire des mentalités, Histoire de la médecine

Keywords : Evliyâ Çelebi (1611-1682), Seyahatnâme, surgery, European medical science, Ottoman Empire, seventeenth century, History of mentalities, travelogues, History of medicine motsclestr Evliyâ Çelebi (1611-1682), Seyahatnâme, Cerrahlık, Avrupalı tıbbi uygulamalar, Osmanlı İmparatorluğu, Onyedinci yüzyılda, Tarih, Zihniyetlerin Tarihi, Seyahatnameler Mots-clés : Evliyâ Çelebi (1611-1682), Evliyâ Çelebi (1611-1682), Seyahatnâme, Seyahatnâme, chirurgie, pratiques médicales européennes, récit de voyage

Index géographique : Empire ottoman

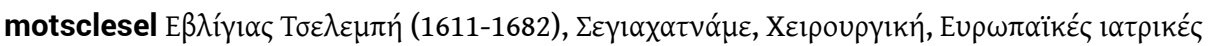

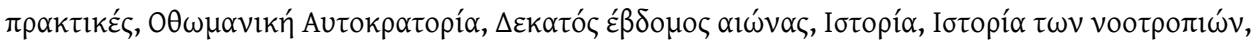

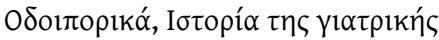

motsclesmk ЕВЛИЈА ЧЕЛЕБИЈА, (1611-1682) СЕЈАХАТНАМЕ, ХИРУРГИЈА, ЕВРОПСКА МЕДИЦИНСКИ ПРАКТИКИ, ОТОМАНСКАТА ИМПЕРИЈА, СЕДУЧИАЕСЕТИОТ ВЕК, ИСТОРИЈА, ИСТОРИА НА МЕНТАЛИТЕТ, ПАТЕПИСИ

Index chronologique : dix-septième siècle

\section{AUTEUR}

\section{LUCA BERARDI}

Professeur

Instituto Universitario, Naples, Italie 